\title{
Detection of internal short circuit in Li-ion battery by estimating its resistance
}

\author{
Minhwan Seo ${ }^{\mathrm{a}}$, Taedong Goh ${ }^{\mathrm{b}}$, Gyogwon Koo ${ }^{\mathrm{a}}$, Minjun Park ${ }^{\mathrm{a}}$ and Sang Woo Kima,**
}

${ }^{a}$ Department of Electrical Engineering, Pohang University of Science and Technology(POSTECH), 77 Cheongam-Ro, Nam-Gu, Pohang, 37673, Korea

${ }^{\mathrm{b}}$ Department of Creative IT Excellence Engineering and Future IT Innovation Laboratory, Pohang University of Science and Technology(POSTECH), 77 Cheongam-Ro, Nam-Gu, Pohang, 37673, Korea

*Corresponding Author: swkim@postech.edu

\begin{abstract}
We propose an algorithm for estimating an internal short circuit (ISC) resistance in a Li-ion battery. With a simple equivalent model of the Li-ion battery and recursive least square (RLS) algorithm, open circuit voltage (OCV) and State of Charge (SOC) are estimated. By using the estimated OCV and SOC, the ISC resistance can be calculated and used as ISC fault index. To verify this algorithm, the simulation data from MATLAB/Simulink model and experiment data are used. The result shows that the proposed algorithm contributes to detect the ISC fault in Li-ion battery by using the new ISC fault index.
\end{abstract}

Keywords: fault diagnosis, safety, parameter estimation.

\section{Introduction}

The Li-ion batteries are popularly used in transportation applications, such as electrical vehicle, hybrid electrical vehicle, and energy storage system because of their high energy and power density ${ }^{(1)}$. However, many dangerous accidents associated with a battery have happened by internal short circuit (ISC), safety concerns always is remained $^{(2,3)}$. This fault increase a temperature of a battery cell and especially the temperature above $100^{\circ} \mathrm{C}$ causes exothermic side reactions, which finally leads to thermal runaway with fire and explosion ${ }^{(4,5,6)}$.

For these reasons, studies for ISC detection are presented. Xia and Bing introduced the method of detecting ISC fault. After experimenting the ISC fault and finding threshold values, such as variation of terminal voltage and temperature and nominal current, ISC are detected ${ }^{(7,8)}$. However, prior experiment about ISC fault must be conducted to find the thresholds values. When ISC happens in a lithium ion battery, the variation of internal parameters used in equivalent circuit model for Li-ion battery are employed for detecting $\mathrm{ISC}^{(9)}$ or for making thresholds values to find out ISC fault ${ }^{(10)}$. However, the introduced methods are verified with one particular current profile, and it is difficult to know a physical meaning of thresholds.

Therefore, we proposed a new algorithm for detecting ISC fault by estimating its resistance which directly calculates the physical value related with the fault. Two current profiles are employed to verify the usefulness of the proposed algorithm.

In the remainder of the paper, the proposed algorithm will be introduced in Section 2, the simulation model of Liion battery and result of simulation will be described in Section 3, and from experiment data, the result of estimating the ISC resistance and discussion will be presented in Section 4.

\section{ISC resistance estimation algorithm}

\subsection{Overview of the proposed algorithm}

When the ISC occurs in a Li-ion battery, the Li-ion battery is discharged not only by the load current and but also by the self-discharge current caused by the ISC. Under the existence of the self-discharge current, the terminal voltage rapidly decreased. In addition, the ISC in the Li-ion battery leads to drop of the OCV and State of Charge (SOC). Compared with an internal resistance (e.g., $50 \mathrm{~m} \Omega$ for 18650 Li-ion battery), the soft shorts have big magnitude of short resistance such as $10 \Omega$ and take a lot of time to initiate the emergency situation like thermal runaway. The more the $\mathrm{Li}$ ion battery is cycled with soft short, soft short in the Li-ion 
battery more severely deteriorates, and causes the thermal runaway consequently ${ }^{(4,10)}$. Therefore it is needed to find out the index for early detecting the ISC fault. To determine existence of the ISC in Li-ion battery, the terminal voltage difference between the normal battery and the ISC fault battery were used ${ }^{(7,8)}$. However, the terminal voltage is affected by measurement noise and load current. Compared with the terminal voltage difference, the differences of OCV and SOC are less influenced by the noise and the load current. The OCV of ISC fault battery can be estimated by using Recursive Least Square (RLS) algorithm with forgetting factor. Then SOC of ISC fault battery is obtained by using a SOC-OCV look up table. After developing equations about $\mathrm{SOC}$, the equation related with self-discharge due to the ISC is derived and the ISC resistance is calculated. We define the estimated ISC resistance as the index and detect the ISC fault if estimated ISC resistance is lower than $10 \Omega$.

\subsection{Equivalent circuit model and Estimation of OCV and SOC}

Tommy ${ }^{(5)}$ and Rui ${ }^{(11)}$ presented that the ISC and external short circuit that a battery is connected with an external resistance in parallel have similar characteristics. The tendencies are that the terminal voltage decreases, temperature in a Li-ion battery rapidly rises and so on. To easily develop the equation about OCV, the simple equivalent circuit model is used. A normal battery model is introduced in Fig.1.(a), where $V_{O C}$ is the normal OCV, $R$ is the normal internal resistance, $I$ is the normal load current and $V_{t}$ is the normal terminal voltage. An ISC fault battery model is presented in Fig.1.(b). Especially $I_{1 f}$ and $\mathrm{I}_{2 f}$ are distributed current by ISC resistance $\left(R_{I S C f}\right)$, and $I_{2 f}$ flows on $R_{I S C f}$ and describes the self-discharge current. To distinguish between normal model parameters and fault model parameters, subscript $f$ is added to all parameters related with fault model.

By Ohm's law the terminal voltage dynamic of normal battery model is explained in (1) and the dynamic of ISC fault model is described in (2) and (3) $)^{(10)}$, where $k$ denotes the discretization step with a sample period T.

$$
\begin{aligned}
& V_{t}(k)=V_{O C}(k)+I(k) R \\
& I_{f}(k)=I_{1 f}(k)+I_{2 f}(k) \\
& V_{t f}(k)=V_{O C f}(k)+R_{f} I_{1 f}(k)
\end{aligned}
$$

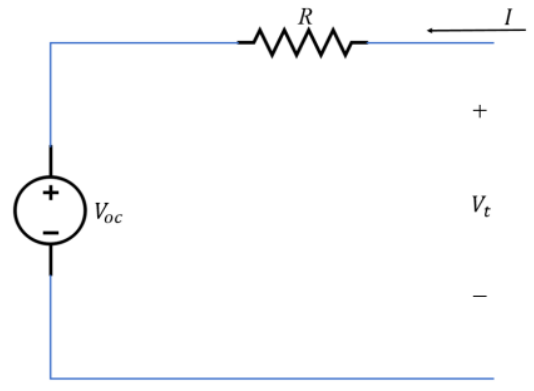

(a) Normal battery model

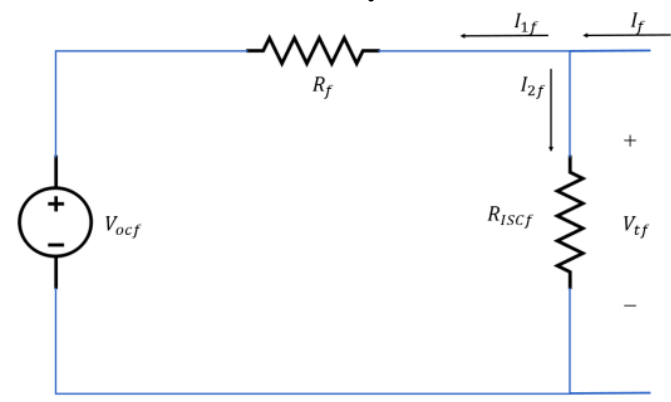

(b) ISC fault battery model

Fig. 1. Equivalent circuit model.

$$
\begin{aligned}
& V_{t f}(k)=\frac{R_{I S C f}}{R_{f}+R_{I S C f}} V_{O C f}(k)+ \\
& \frac{R_{f} R_{I S C f}}{R_{f}+R_{I S C f}} I_{f}(\mathrm{k})
\end{aligned}
$$

It is possible to derive RLS equations for normal battery which is described in (4) and for ISC fault battery which is described in (5) separately ${ }^{(12)}$.

$$
\begin{aligned}
& y_{1}=V_{t}(k)=\theta_{1}^{T} \emptyset_{1} \\
& \emptyset_{1}=[1, I(k)] \\
& \theta_{1}=\left[V_{O C}(k), R\right] \\
& y_{2_{f}}=V_{t f}(k)=\theta_{2 f}^{T} \emptyset_{2 f} \\
& \emptyset_{2 f}=\left[1, I_{f}(k)\right] \\
& \theta_{2 f} \\
& =\left[\frac{R_{I S C f}}{R_{f}+R_{I S C f}} V_{O C f}(k), \frac{R_{f} R_{I S C f}}{R_{f}+R_{I S C f}}\right]
\end{aligned}
$$

Using RLS algorithm with (4) and (5), the values of OCV can be estimated as first elements of $\theta_{1}$ and $\theta_{2 f}$. Because the ISC resistance is much bigger than the internal resistance in an initial stage of ISC, $\frac{R_{I S C f}}{R_{f}+R_{I S C f}} V_{O C f}(k)$ can be approximated to $V_{O C f}(k)$. Using the MATLAB/Simulink model which will be explained in section 3.1, the estimated $\mathrm{OCV}$ values are obtained and described in Fig.2 depending on ISC resistance magnitudes. The smaller the ISC resistance 


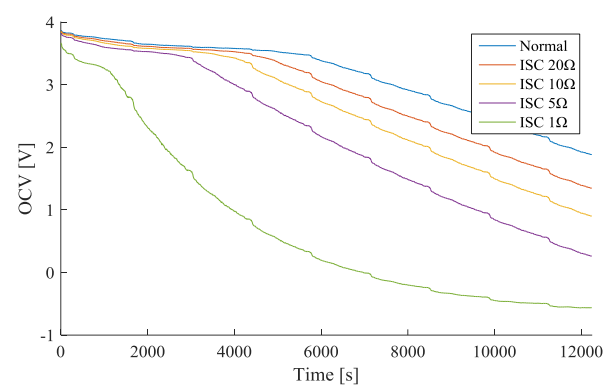

Fig. 2. Difference between estimated OCVs.

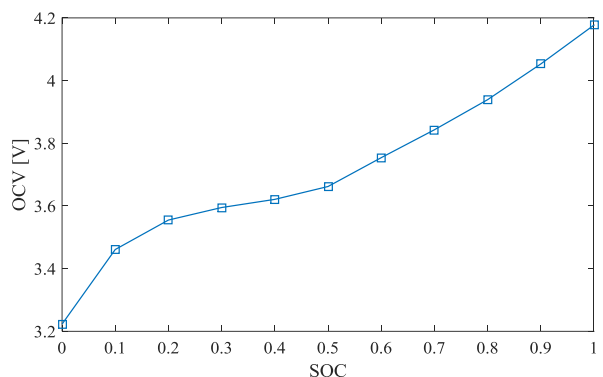

Fig. 3. SOC-OCV curve.

is, the bigger the difference between normal OCV and ISC fault OCV is

We implemented an experiment to get the look-up table described in Fig.3. A constant current (0.5C) was included in full charged Li-ion battery (INR18650-20R, 2Ah) during 12 minutes to decrease the $10 \%$ of SOC. Then the battery was put on the inactive status during 60 minutes to measure the $\mathrm{OCV}$. After repeating the experiment, we obtained the OCV depending on the SOC that is SOC-OCV curve. Using the SOC-OCV curve the SOC values can be obtained, and also the fact that difference between normal SOC and ISC fault SOC tends to be similar with the difference from the OCV case is checked according to Fig.4.

\subsection{Estimations of ISC resistance}

The coulomb counting method is commonly used and reliable to calculate the SOC if the load current and the initial SOC value are known. The method with discretization step is followed as (6).

$$
\begin{aligned}
& S O C_{f}(k)=S O C_{f}(0)+ \\
& \frac{T}{C_{\max }} \sum_{n=1}^{k}\left[I_{f}(n)-I_{2 f}(n)\right]
\end{aligned}
$$

where $T$ is the sample rate $(0.1 \mathrm{~s})$ and $C_{\max }$ is the maximum capacity of the battery $(2.0599 A h)$.

After adding $\operatorname{SOC}(k+1)$ and $\operatorname{SOC}(k)$, the result is described in (7) and consists of the first term about load current and second term about self-discharge current. The self-discharge current can be replaced with $I_{2 f}=\frac{V_{t f}}{R_{I S C f}}$.

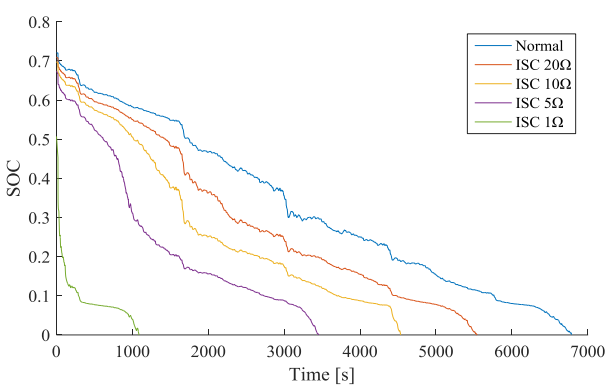

Fig. 4. Difference between estimated SOCs.

$$
\begin{aligned}
& \operatorname{SOC}_{\mathrm{f}}(k+1)-S O C_{f}(k)= \\
& \frac{T}{C_{\max }} I_{f}(k+1)- \\
& \frac{T}{C_{\max }} \frac{1}{R_{I S C f}} V_{t f}(k+1) \\
& S O C_{f}(k)-S O C_{f}(1)= \\
& \frac{T}{C_{\max }}\left[I_{f}(k)+I_{f}(k-1)+\cdot .\right. \\
& \left.\cdot+I_{f}(2)\right]-\frac{T}{C_{\max }} \frac{1}{R_{I S C f}}\left[V_{t f}(k)+\right. \\
& \left.V_{t f}(k-1)+\cdots+V_{t f}(2)\right]
\end{aligned}
$$

Because it is difficult to see the variation of SOC in a one step, the estimated ISC resistance is incorrect. According to (8), the step between initial SOC value and $k$ th SOC value can be spanned. Then the left term of (8) can be calculated after getting the SOC values. With the values $\left(C_{\text {max }}, T\right)$ and measured values $\left(I_{f}, V_{t f}\right)$, the unknown value $\left(R_{I S C f}\right)$ can also calculated from simulation or experiment data.

\section{Simulation of ISC battery}

\subsection{MATLAB/Simulink model}

The MATLAB/Simulink is the popular method to make a simulation model of application because of convenient and user friendly tool box provided in MATLAB. For this reason Simulink models of Li-ion battery already have been introduced $^{(13,14)}$. In this paper, simply Simulink model

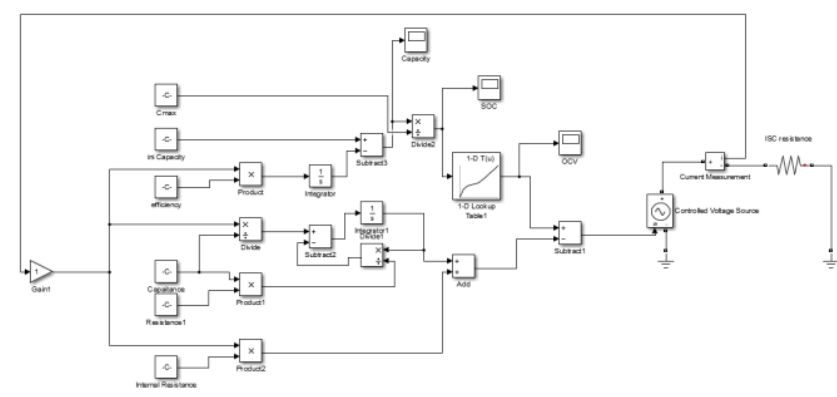

Fig. 5. MATLAB/Simulink model. 


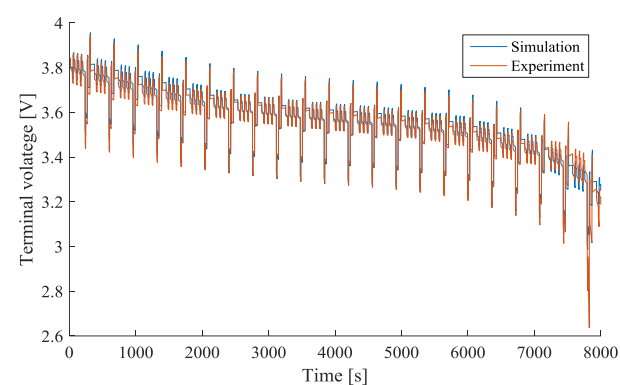

Fig. 6. Terminal voltage.

consists of constant parameters which are internal resistance $(57 \mathrm{~m} \Omega)$ and RC network parameters $(23 \mathrm{~m} \Omega, 937 \mathrm{~F})$ according to Fig.5. The terminal voltage of simulation is very similar with it of experiment (Fig.6).

\subsection{Simulation results and discussion}

The Dynamic Stress Test (DST) and Urban Dynamometer Driving Schedule (UDDS) are used as input load current to verify that the proposed algorithm can estimate the ISC resistance in varied environment. The maximum value of these profiles is $5 \mathrm{~A}$ with sample rate $0.1 \mathrm{~s}$, and an initial SOC is 70\%. To use RLS algorithm, the forgetting factor is 0.999 for this paper. When the applied load current are DST and UDDS, the results of estimated ISC resistance depending on $20 \Omega, 10 \Omega, 5 \Omega$ and $1 \Omega$ are shown in Fig.7. The final values of Fig.7 are together presented in Table.1. Although there are some errors, the estimated results are similar with the real values and considerably accurate.

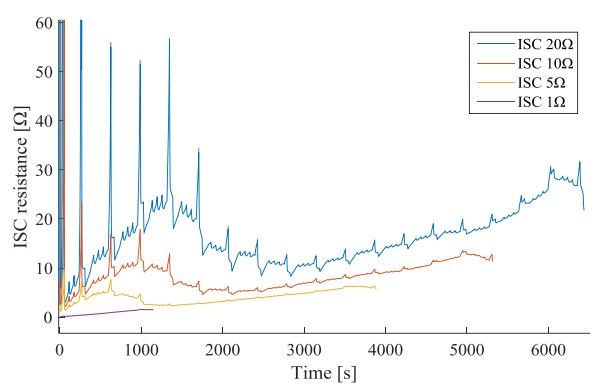

(a) DST profile is applied

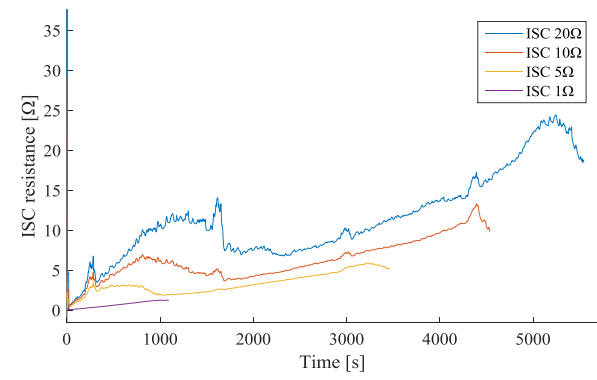

(b) UDDS profile is applied

Fig. 7. Estimated ISC resistances in simulation
Table 1. The results of the simulation.

\begin{tabular}{|c|c|c|c|c|}
\hline ISC resistance & $1 \Omega$ & $5 \Omega$ & $10 \Omega$ & $20 \Omega$ \\
\hline DST & $1.5 \Omega$ & $5.8 \Omega$ & $11.4 \Omega$ & $21.8 \Omega$ \\
\hline UDDS & $1.24 \Omega$ & $5.22 \Omega$ & $9.87 \Omega$ & $18.6 \Omega$ \\
\hline
\end{tabular}

\section{Experiment}

\subsection{ISC experiment}

An ISC experiment consists of one Li-ion battery and ISC resistance described in Fig.8. The load currents are same with them used in simulation, and the resistance $20 \Omega, 10 \Omega$, $5 \Omega$ and $1 \Omega$ are employed to present ISC fault in Li-ion battery. The initial SOC of Li-ion battery is $70 \%$. A Chroma battery charge/discharge system is used to give the load current and to measure the terminal voltage with sample rate $0.1 \mathrm{~s}$.

\subsection{Experiment results and discussion}

The ISC resistances respectively for DST profile and UDDS profile are estimated and described in Fig.9. The final values of estimated ISC resistance are arranged in Table.2.

Compared with the results of simulation, the estimated values of ISC resistance $20 \Omega$ are not accurate. When the ISC resistance is larger $10 \Omega$ there is small difference between normal SOC and faulted SOC, and the measurement noise can't be ignored. Thus, it is difficult to accurately estimate the ISC resistance. However, the purpose of this paper is not to find exactly value of ISC resistance but to notify the existence of ISC fault in battery by defining ISC resistance as the fault index. Therefore we can give the alarm of ISC fault to user if this proposed algorithm find out the ISC resistance which is smaller than $10 \Omega$.

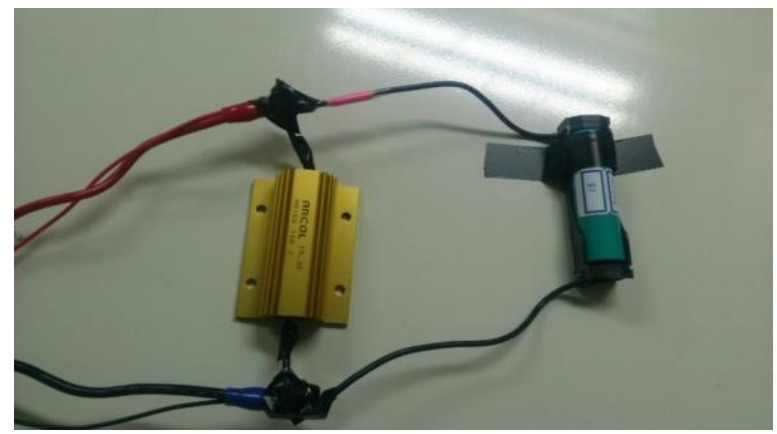

Fig. 8. ISC experiment 


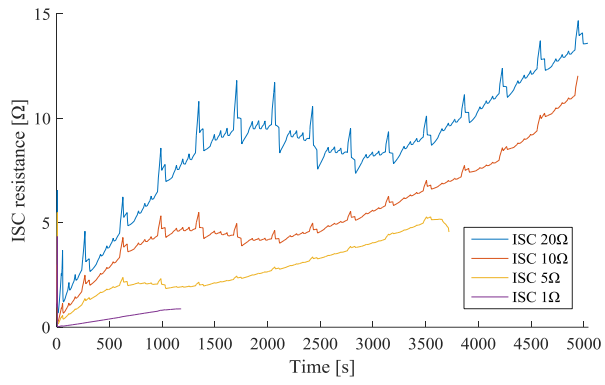

(a) DST profile is applied

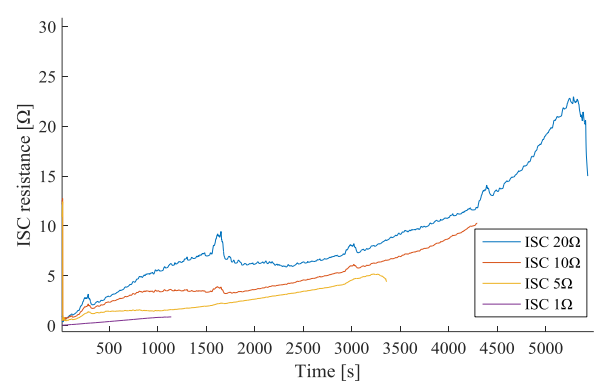

(b) UDDS profile is applied

Fig. 9. Estimated ISC resistances in experiment

Table 2. The results of the experiment.

\begin{tabular}{|c|c|c|c|c|}
\hline IsC resistance & $1 \Omega$ & $5 \Omega$ & $10 \Omega$ & $20 \Omega$ \\
\hline DST & $0.88 \Omega$ & $4.5 \Omega$ & $12.0 \Omega$ & $13.6 \Omega$ \\
\hline UDDS & $0.86 \Omega$ & $4.4 \Omega$ & $10.3 \Omega$ & $14.9 \Omega$ \\
\hline
\end{tabular}

Errors increase as ISC resistance goes to small value $1 \Omega$ since the error from the assumption, which is that the ISC resistance is much bigger than the internal resistance defined in chap2, is unable to be neglected. However, the ISC fault can be early detected at big value of ISC resistance by this proposed algorithm before a battery has small ISC resistance about $1 \Omega$.

\section{Conclusion}

In this paper the ISC estimation algorithm determining existence of the ISC fault in Li-ion battery is introduced. Without conducting prior ISC experiment for defining the thresholds, the proposed algorithm can detect the ISC fault by directly estimating the ISC resistance as the fault index. To estimate the faulted OCV and SOC, the simple equivalent circuit model and RLS algorithm with forgetting factor are used and then the estimated ISC resistance are calculated. Two current profiles are employed to verify the proposed algorithm, and ISC fault can be consistently detected by the proposed algorithm.

Our future research will concentrate on to improve the accuracy of estimating ISC resistance and to extend the verification area such as several of current profiles and battery pack.

\section{Acknowledgment}

"This research was supported by the MSIP (Ministry of Science, ICT and Future Planning), Korea, Under the "ICT Consilience Creative Program" (IITP-R0346-16-1007) supervised by the IITP (Institute for Information \& communications Technology Promotion)"

\section{References}

(1) M. Stanley Whittingham : "Materials Challenges Facing Electrical Energy Storage", MRS Bulletin, 33, pp. 411419, 2008

(2) Aircraft incident report: auxiliary power unit battery fire. Japan airlines Boeing 787, JA 829J, Boston, Massachusetts, January 7, 2013. National Transportation Safety Board, DC. Rep no. PB2014108867; November 21, 2014

(3) http://www.camxpower.com/wpcontent/uploads/46.pdf -Lithium-Ion Battery Safety: Detection of Developing Internal Shorts and Suppression of Thermal Runaway

(4) Zhang, Jingliang and Jay Lee : "A review on prognostics and health monitoring of Li-ion battery.", Journal of Power Sources, 196.15, pp. 6007-6014, 2011

(5) Zavalis, Tommy Georgios, Mårten Behm, and Göran Lindbergh : "Investigation of short-circuit scenarios in a lithium-ion battery cell.", Journal of The Electrochemical Society, 159.6, pp A848-A859, 2012

(6) Spotnitz, R., and J. Franklin : "Abuse behavior of highpower, lithium-ion cells.", Journal of Power Sources, 113.1,pp. 81-100, 2003

(7) Xia, Bing, et al : "Multiple cell lithium-ion battery system electric fault online diagnostics.", Transportation Electrification Conference and Expo (ITEC), 2015

(8) Xia, Bing, et al : "External short circuit fault diagnosis for lithium-ion batteries.", Transportation Electrification Conference and Expo (ITEC), 2014

(9) Feng, Xuning, et al : "Online internal short circuit detection for a large format lithium ion battery.", Applied Energy, 161, pp. 168-180, 2016

(10) Ouyang, Minggao, et al : "Internal short circuit detection for battery pack using equivalent parameter and consistency method.", Journal of Power Sources, 
294, pp. 272-283, 2015

(11) Zhao, Rui, Jie Liu, and Junjie Gu : "Simulation and experimental study on lithium ion battery short circuit.", Applied Energy, 173, pp. 29-39, 2016

(12) He, Hongwen, et al : "Online model-based estimation of state-of-charge and open-circuit voltage of lithiumion batteries in electric vehicles.", Energy, 39.1, pp. 310318, 2012

(13) Yao, Low Wen, et al : "Modeling of lithium-ion battery using MATLAB/Simulink.", IECON 2013-39th Annual Conference of the IEEE, 2013

(14) M. Knauff, J. McLaughlin, C. Dafis, D. Niebur, H. Kwatny, C. Nwankpa,and J. Metzer : "Simulink Model of a Lithium-ion Battery for the Hybrid Power System Testbed", Proceedings of the ASNE Intelligent Ships Symposium, 2007 